FUNDACIÓN KOINONIA (F.K). Santa Ana de Coro. Venezuela.

Miriam Viviana Mora-Bojorque; Luis Bolívar Cabrera-Berrezueta; Nelson Cristóbal Reascos-Vallejo Roxanna Auccahuallpa-Fernández

http://dx.doi.org/10.35381/r.k.v6i4.1504

\title{
Storytelling como estrategia para prevenir el bullying
}

\section{Storytelling as a strategy to prevent bullying}

Marco Vinicio Deleg-Muzha

marco.deleg.71@est.ucacue.edu.ec

Universidad Católica de Cuenca, Azogues

Ecuador

https://orcid.org/0000-0001-6808-3808

Sergio Constantino Ochoa-Encalada

scochoae@ucacue.edu.ec

Universidad Católica de Cuenca, Cuenca

Ecuador

https://orcid.org/0000-0003-3067-3719

Diego Eduardo Apolo-Buenaño

diego.apolo@ucacue.edu.ec

Universidad Católica de Cuenca, Cuenca

Ecuador

https://orcid.org/0000-0002-1123-1483

Juan Pablo Pazmiño-Piedra

juan.pazmino@ucacue.edu.ec

Universidad Católica de Cuenca, Cuenca

Ecuador

https://orcid.org/0000-0003-0069-7680

Recepción: 30 de agosto 2021

Revisado: 20 de septiembre 2021

Aprobación: 15 de noviembre 2021

Publicación: 01 de diciembre 2021 
Revista Arbitrada Interdisciplinaria KOINONIA

Año VI. Vol VI. N4. Edición Especial: Educación III. 2021

Hecho el depósito de Ley: FA2016000010

ISSN: 2542-3088

FUNDACIÓN KOINONIA (F.K). Santa Ana de Coro. Venezuela.

Miriam Viviana Mora-Bojorque; Luis Bolívar Cabrera-Berrezueta; Nelson Cristóbal Reascos-Vallejo

Roxanna Auccahuallpa-Fernández

\title{
RESUMEN
}

El objetivo de esta investigación fue analizar el uso del Storytelling como estrategia que puede ayudar a prevenir el bullying en la institución educativa American School, para la cual se realizó una investigación de carácter descriptiva no experimental de cohorte transversal. Los alumnos consideran que la prevención del acoso escolar debe ser parte del plan de estudios de la institución y que no están de acuerdo ni en desacuerdo que el uso de los medios digitales son una herramienta para la prevención del acoso. Con esto se abre la posibilidad de poder utilizar el storytelling como estrategia para la prevención del acoso escolar debido a que al momento de crear las historias los estudiantes se volverán parte de la misma, se interrelacionaran y trabajaran en equipo fomentando los valores de la empatía y el respeto entre ellos, además de conectar emocionalmente con el mensaje que desean transmitir.

Descriptores: Acoso; relaciones entre pares; ambiente educacional. (Palabras tomadas del Tesauro UNESCO).

\begin{abstract}
The objective of this research was to analyze the use of Storytelling as a strategy that can help prevent bullying at the American School educational institution, for which a descriptive, non-experimental cross-sectional cohort investigation was carried out. Students consider that bullying prevention should be part of the institution's curriculum and that they neither agree nor disagree that the use of digital media is a tool for bullying prevention. This opens the possibility of being able to use storytelling as a strategy for the prevention of school bullying because at the moment of creating the stories the students will become part of it, interrelate and work as a team promoting the values of empathy and the respect between them, in addition to connecting emotionally with the message they want to convey.
\end{abstract}

Descriptors: Bullying; peer relationship; educational environment. (Words taken from the UNESCO Thesaurus). 


\section{INTRODUCCIÓN}

El comportamiento intimidante y agresivo, así como la violencia y el acoso escolar son fenómenos que se están volviendo cada vez más frecuentes en instituciones de educación. Afectan en gran medida, la actitud y el comportamiento de los estudiantes, el desarrollo psicoemocional y su rendimiento escolar, también puede conducir a un trauma psicológico grave y un comportamiento disfuncional es importante reconocer que la víctima es más débil e impotente en comparación con el abusador. Los ataques están programados, en repetidos a intervalos de forma regular y el objetivo del abusador es la afirmación de su poder sobre la víctima (Fokides, 2017).

La violencia escolar, como fenómeno social, tiene una estrecha relación con la discriminación social, la exclusión social y la diversidad cultural. En general la ocurrencia del bullying en las escuelas y colegios son más bajas que en otros países, sin embargo, se vuelven cada vez más frecuentes, estos episodios durar un período de tiempo breve, el $19 \%$ de incidentes puede durar más de un año, cabe indicar que los problemas de violencia y acoso actualmente son considerados como problemas de salud pública por el hecho de la influencia en su bienestar y desarrollo psicológico (Valdés-Cuervo et al. 2018).

En una situación de acoso, las personas asumen uno de los siguientes roles; acosadores y víctimas. Si bien la pareja intimidante-víctima es bastante evidente, la participación de los espectadores es ambiguo, estos espectadores pueden asumir los siguientes roles; tomar activamente partido para intimidar y convertirse en una fuente de poder para el intimidador, es decir tomando una posición de soporte, o, de forma pasiva, al observar y, con frecuencia, al guardar silencio aprobar la conducta y acciones del agresor. Por último, también el espectador puede ayudar a la víctima o pedir a un adulto que intervenga en el conflicto (Tsai et al. 2011). 
Según varios autores, además señalan que en América Latina la prevalencia de la violencia y/o acoso escolar en múltiples formas es agravante, analizando el caso de estudio de la Unicef asociada en Latinoamérica proporciona datos de que el 50 y $70 \%$ de alumnos han sido envueltos en casos de agresión entre compañeros, así también analizando 16 países; en este sentido, (Castillo-Pulido et al. 2011), señalan de que los valores de acoso llegan al $63 \%$ de la población escolar, esto indica que de cada tres estudiantes uno está involucrado en un episodio de acoso y dentro del ámbito del ciberbullying uno de cada cinco (Herrera-López et al. 2018).

Por otra parte, a nivel nacional, la problemática abordada es foco de interés ya que, a pesar de contar con investigaciones de carácter sobre la violencia escolar, no existen estudios de forma integral, ni tampoco ha sido estudiado de forma específica, según los datos analizados por el Ministerio de Educación de Ecuador se tienen cifras de que 6 de cada 10 alumnos en un rango de edad entre 11 a 18 años, han sido víctimas de acoso escolar analizando una muestra de caso 5500 estudiantes (Vargas-Martínez et al. 2018). Teniendo en cuenta la magnitud del desarrollo del problema, asociado a que el bullying y el ciberbullying son un problema globalizado y de poca detección se plantea la estrategia del uso del Storytelling para mejorar estas situaciones y poder garantizar un ambiente apto para el desarrollo de los estudiantes.

La narrativa ha demostrado, que la narrativa y la historia de la narración son útiles para mejorar el pensamiento de los estudiantes en el proceso educativo. La narración permite a los estudiantes presentar una mejor estructura del pensamiento y proporcionar de manera óptima un mejor desarrollo del pensamiento abstracto. Por otra parte, la creación de la animación multimedia interactiva, es interesante para los estudiantes (McLellan, 2006). La narración mediante la realización de una animación, o conocido también como storytelling tiene como objetivo, motivar a los estudiantes a participar en el aprendizaje e involucrarlos en actividades de aprendizaje. Por ello actualmente se considera una 
herramienta interactiva e innovadora como estrategia de prevención e información con varios ámbitos de aplicación.

La presente investigación tiene como objetivo principal, analizar de qué manera el uso del storytelling como estrategia puede ayudar a prevenir el bullying en la institución educativa American School, ubicado en la provincia del Azuay, cantón Cuenca, con el fin de captar e involucrar a los estudiantes desde lo emocional mediante el uso de herramientas digitales como audio, video e interactividad, así de esta manera resaltar y promover los valores del respeto, empatía con el propósito de una convivencia armónica entre pares, debido a que las estrategias que actualmente son implementadas ya no tienen un efecto positivo, esto se debe a la utilización de nuevas herramientas digitales por parte de los estudiantes y por ende de nuevos canales de difusión a los cuales están más seducidos y consumen mayor información (Fokides, 2017).

Al mencionar storytelling básicamente nos referimos a las historias contadas de una manera distinta, en donde el mensaje es fácil de recordar debido a que puede construir relaciones entre el espectador de situaciones vividas, una correcta estructura de narrativa estimulara las emociones provocando el interés y un nexo con el público, así mismo el escenario o imágenes utilizadas en el relato mantendrán la atención y despertarán las emociones del espectador.

Las historias son experiencias estructuradas en una unidad coherente, con la intención de unificar acciones y eventos en formas que crean significado. Además, en contraste para el storytelling, las historias son egoístas, en el sentido de que los individuos cuentan historias con el fin de aclarar su propia posición en el mundo, en otras palabras, para crear un significado personal en relación con una narrativa más grandiosa.

Así, por ejemplo, se sugieren emociones a una persona para informarla de la relación entre ella misma y un contexto más amplio, mediante la herramienta de storytelling. Por lo tanto, contar historias puede ser una forma de procesar las emociones. Los individuos pueden procesar historias de manera adaptativa o desadaptativa, en la primera conduce 
a un mayor sentimiento de aceptación y comprensión mientras que en la segunda se exacerba un sentimiento de angustia (Mathisen, 2019).

Por otra parte, autoestima es omnipresente en la vida contemporánea. En aulas y lugares de trabajo, eventos deportivos y recitales de música, la gente generalmente asume que la autoestima es fundamental para el éxito en ese dominio. Por supuesto, la promoción de la autoestima y la prevención de la baja la autoestima, se percibe ampliamente como un importante factor social, es un objetivo que amerita intervenciones generalizadas para impulsar los niveles de autoestima en la población. La autoestima se refiere a la evaluación subjetiva de un individuo de su valor como persona (Orth \& Robins, 2014). Es importante destacar que la autoestima no refleja necesariamente unos talentos y habilidades objetivos de la persona, o incluso cómo la perspectiva de la persona es evaluada por otros. Además, la autoestima es comúnmente conceptualizada como el "sentimiento de que uno es suficientemente bueno" y, en consecuencia, las personas con alto autoestima no necesariamente creen que son superiores a otros. Por tanto, la autoestima implica sentimientos de auto aceptación y auto respeto, en contraste con la excesiva autoestima y auto engrandecimiento que caracteriza a los individuos narcisistas.

La autoestima tiene una relación directa con la psicología, por ello es que las relaciones sociales juegan un papel clave en la configuración de la autoestima en las personas o en la evaluación subjetiva de su valía general como persona. Aunque existe un gran apoyo empírico para la asociación concurrente entre las diversas características de la relación y autoestima (Harris \& Orth, 2020).

Dentro de las investigaciones relacionadas a la utilización de storytelling como mecanismo para la reducción de bullying se presenta el estudio de (Puspitasari et al. 2018), estudio que se produjo en Indonesia, en un centro educativo ubicado en MI Manbahul Ulum, el mismo, el cual se implementó un proceso educativo, para que se familiaricen y contribuir en el proceso de prevención del acoso entre el niños, en este 
estudio se tomó una muestra de 20 niños y niñas de edad escolar primara de una zona rural, estos participaron en el proyecto de una edad similar pero de un origen social diferente.

En el proyecto se presentó a los niños la temática de bullying y ciertas historias asociadas al tema, cabe indicar que la mayoría de niños son bilingües, este estudio se realizó por dos meses de junio a agosto de 2018, en el cual los niños entendieron por medio de la herramienta storytelling que la intimidación es un hecho inhumano y que pueden tomar la decisión de no hacerlo, una etapa relevante fue la etapa de en el proceso de desarrollo de la narración apoyado por canciones, capturó puntos relevantes de la letra, la misma que se refiere a la escucha activa, corroborando que la escucha activa puede fomentar la alfabetización (Puspitasari et al. 2018).

Para mejorar la eficacia del aprendizaje, se realizó en una escuela secundaria pública en Grecia, este proyecto tuvo una duración de tres años en donde se realizaron tres mecanismos de intervención: piloto, una intervención principal y una intervención iterativa, en este estudio tanto profesores, así como estudiantes participaron en la creación de historias digitales, ya que la herramienta de storytelling se utilizó como una instrucción y como una herramienta de aprendizaje (Chautard \& Collin-Lachaud, 2019). La herramienta de storytelling, se implementó en tres clases del primer grado de la escuela secundaria y se trabajó en grupos de 4 a 5 personas, se utilizaron movimientos literarios como romanticismo, simbolismo, modernismo y surrealismo aplicado al bullying para entender las distintas perspectivas con cuatro enfoques. En conclusión, fueron capaces de articular su propia voz, de ejercitar sus habilidades de lectura y semiótica, experimentando con la dinámica de una herramienta nueva en la que por medio de la palabra y explorando las múltiples cargas multimedia, y finalmente tomar un papel más activo y empoderador, yendo más allá de la posición del estudiante - lector en la posición del estudiante - escritor, actualizando su punto de vista y finalmente mejorando sus perspectivas y una postura firme en relación al abuso escolar. 
Revista Arbitrada Interdisciplinaria KOINONIA

Año VI. Vol VI. N4. Edición Especial: Educación III. 2021

Hecho el depósito de Ley: FA2016000010 ISSN: $2542-3088$

FUNDACIÓN KOINONIA (F.K). Santa Ana de Coro. Venezuela.

Miriam Viviana Mora-Bojorque; Luis Bolívar Cabrera-Berrezueta; Nelson Cristóbal Reascos-Vallejo

Roxanna Auccahuallpa-Fernández

Por otro lado, el bullying es considerado como un comportamiento agresivo que sigue un patrón y que es repetitivo, violento y con intencionalidad ya que se manifiesta en un contexto donde exista desigualdad de autoridad o fuerza entre los individuos. Este tipo de violencia se da de forma grupal en medio de la interrelación entre estudiantes, se manifiesta entre los niños en edad escolar que implica un desequilibrio de poder real o percibido por los estudiantes, tiene un alto potencial de repetirse, con el tiempo. Tanto los niños que son acosados como los que acosan a otros niños, pueden tener problemas graves y duraderos (Jiang et al. 2020).

Por otro lado, el uso del storytelling por medio de las imágenes, la imagen como tal, recrea un suceso contado por alguna persona de forma directa o indirecta, desde un elemento fijo de juicio, este tiene la capacidad de transmitir un mensaje por ella, al conocer que una narrativa tiene una imagen fija, está limitada por el conocimiento y el contexto en el que lo perciba el lector, así como referirse directamente a una imagen narrativa da la perspectiva de ser un relato icónico y audiovisual.

Con referencia al uso del discurso en un storytelling, tiene como objeto contar un hecho y está compuesta por anécdotas, leyendas etc. Dentro de la lengua el discurso es la característica de mayor uso dentro de la historia ya que tiene un contexto histórico trascendental iniciado con epopeya, relatos, con la novela. En contraposición el uso de recursos como videos, cambia de forma importante la percepción de la audiencia al establecer un criterio similar proyectado, así también trata de organizarse en secuencia para poder continuar con la narrativa acorde al objetivo planteado a lograr (BuenañoPico, 2016).

Dentro de las características de la utilización de storytelling se tiene que esta es una herramienta que potencia a niños, en los aspectos asociados a la creatividad y el empoderamiento, a partir de esto proyectar ideas a un público determinado, que, mediante la explicación de narraciones digitales usadas en educación, los niños como aprendices tienen la oportunidad de contar una historia o como el componente narrativo 
Revista Arbitrada Interdisciplinaria KOINONIA

Año VI. Vol VI. N4. Edición Especial: Educación III. 2021

Hecho el depósito de Ley: FA2016000010 ISSN: 2542-3088

FUNDACIÓN KOINONIA (F.K). Santa Ana de Coro. Venezuela.

Miriam Viviana Mora-Bojorque; Luis Bolívar Cabrera-Berrezueta; Nelson Cristóbal Reascos-Vallejo

Roxanna Auccahuallpa-Fernández

de una actividad de aprendizaje que tiene la función de transmitir conceptos y mensajes concretos.

Actualmente con la disponibilidad de las redes sociales y la utilización del internet, las historias digitales creadas mediante storytelling se pueden cargar en internet para que este recurso llegue a miles de audiencias, esto ha generado que los niños compartan su comprensión sobre el concepto de acoso escolar desde su punto de vista (Wu \& DerThanq, 2020), considerando su sentir y cuál es el mensaje que quieren transmitir, los niños pueden crear historias digitales y desarrollar sus habilidades de comunicación, aprendiendo a organizar sus ideas, hacer preguntas, expresar opiniones y construir narrativas. En resumen, el storytelling contribuye de una manera positiva para que los niños relacionen el tema a su propia creación de texto, para expresar su voz y pensamiento para una posterior presentación (Puspitasari et al. 2018).

Esto establece que el estado ecuatoriano en materia de derecho y cumplimiento de la constitución debe asegurar y precautelar la salud y salud mental de los niños, niñas y adolescentes del país y determinar los mecanismos de detener el atropello a sus derechos, en este caso considerándose vulnerados por sus pares, sus compañeros.

\section{METODOLOGÍA}

Para el desarrollo de este estudio se realizó una investigación de carácter descriptiva no experimental basada en el objetivo analizar de qué manera el uso de la estrategia del Storytelling puede contribuir a reducir el bullying en la institución educativa American School, con el fin de captar e involucrar a los estudiantes desde lo emocional utilizando herramientas digitales. La muestra poblacional fue aleatoria y estuvo conformada de 38 estudiantes de edades comprendidas entre 16 y 17 años correspondiente al segundo de bachillerato de la Unidad Educativa American School, ubicada en la Provincia del Azuay, cantón Cuenca, sector Capulispamba. 
La técnica que se empleo fue la encuesta con un enfoque cuantitativo de cohorte transversal y un instrumento de tipo cuestionario mediante un escalamiento de Likert, de cinco alternativas de respuestas misma que fue validada mediante el coeficiente de fiabilidad Alfa de Cronbach de 0.717 , calculo que se obtuvo del software SPSS versión 19. Los datos obtenidos fueron procesados mediante estadistica descriptiva.

\section{RESULTADOS}

Los datos obtenidos fueron analizados mediante la prueba de normalidad obteniendo datos paramétricos de acuerdo a Shapiro-Wilk. También se utilizó el análisis de frecuencias para la comprensión de la investigación y por último la prueba del chicuadrado para cruzar variables y conseguir una relación. Luego del análisis de los datos obtenidos en la encuesta se presenta los siguientes resultados:

\section{Tabla 1.}

Los educadores/as juegan un papel importante en la prevención del bullying.

\begin{tabular}{llcccc}
\hline & Frecuencia & Porcentaje & $\begin{array}{c}\text { Porcentaje } \\
\text { válido }\end{array}$ & $\begin{array}{c}\text { Porcentaje } \\
\text { acumulado }\end{array}$ \\
\hline Válidos & Muy de acuerdo & 10 & 26,3 & 26,3 & 26,3 \\
& De acuerdo & 15 & 39,5 & 39,5 & 65,8 \\
& Neutral & 7 & 18,4 & 18,4 & 84,2 \\
& En desacuerdo & 1 & 2,6 & 2,6 & 86,8 \\
& Muy en desacuerdo & 5 & 13,2 & 13,2 & 100,0 \\
\cline { 2 - 6 } & Total & 38 & 100,0 & 100,0 & \\
\hline
\end{tabular}

Fuente: Encuesta.

En la tabla 1 se observa que el $65,8 \%$ de los estudiantes señalan que los educadores juegan un papel importante en la prevención del bullying, lo cual evidencia la importancia de tratar estos temas y generar espacios para consultas. Evidenciando que se puede utilizar nuevas estrategias para dar a conocer estos temas de una manera distinta a las tradicionales. 


\section{Tabla 2.}

Relación entre la prevención del acoso debe ser un plan de la institución y el uso de los medios digitales son una herramienta de prevención.

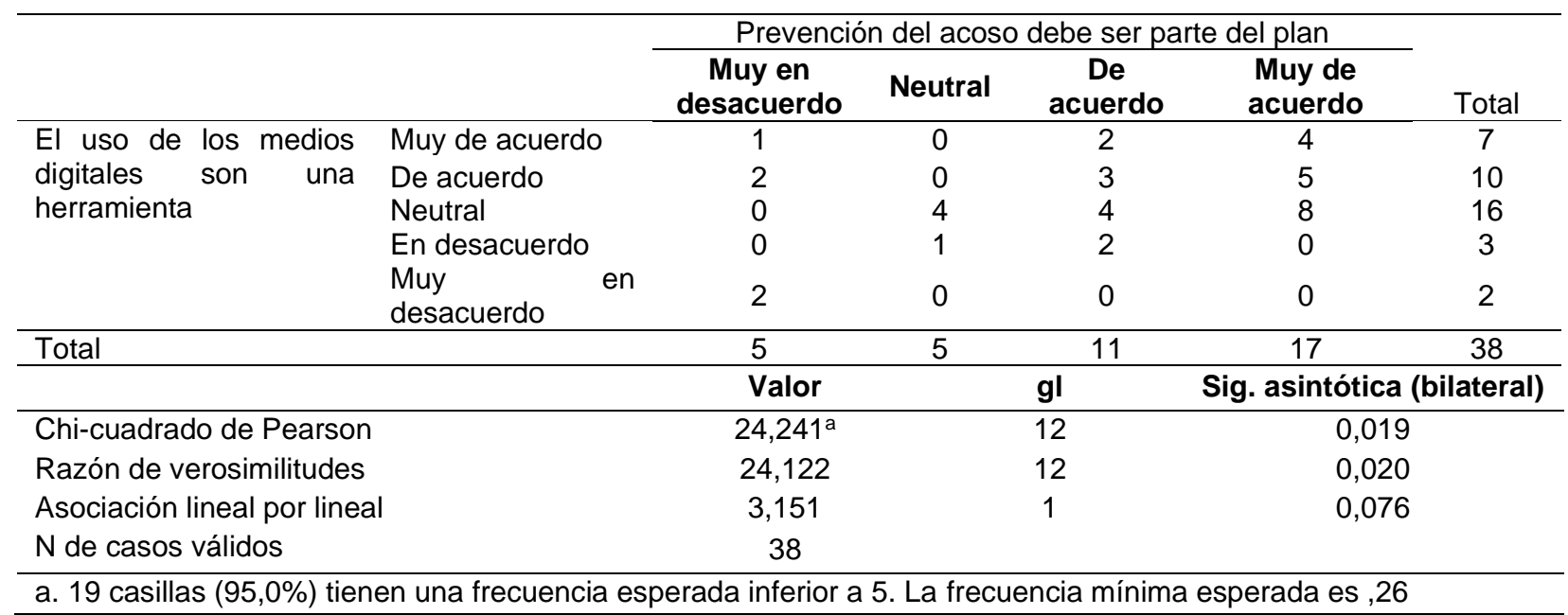

Fuente: Encuesta.

Los resultados de la tabla 2 demuestran que 4 estudiantes están muy de acuerdo que la prevención del acoso deber ser parte del plan de estudios, mientras que 1 de los estudiantes encuestados menciona que está muy de acuerdo que el uso de los medios digitales son una herramienta de prevención del acoso escolar. Con esto podemos concluir que el uso de los medios digitales no está contribuyendo en su gran manera a prevenir el acoso ya sea por distintos factores, como ver los videos o spots publicitarios de siempre, la misma publicidad que no causa o genera ninguna reacción y con eso se pretende decir que no hay una evolución en buscar nuevas herramientas para cautivar a los estudiantes.

Al analizar el coeficiente de relación de Chi-cuadrado de Pearson se evidencia que las variables, la prevención del acoso y el uso de medios digitales no están relacionadas, razón por la cual es posible proponer el uso de una nueva herramienta que es el Storytelling como estrategia para prevenir el bullying. 


\section{Tabla 3.}

El uso de los medios digitales son una herramienta de prevención del acoso escolar.

\begin{tabular}{llcccc}
\hline & Frecuencia & Porcentaje & $\begin{array}{c}\text { Porcentaje } \\
\text { válido }\end{array}$ & $\begin{array}{c}\text { Porcentaje } \\
\text { acumulado }\end{array}$ \\
\hline Válidos & Muy de acuerdo & 7 & 18,4 & 18,4 & 18,4 \\
& De acuerdo & 10 & 26,3 & 26,3 & 44,7 \\
& Neutral & 16 & 42,1 & 42,1 & 86,8 \\
& En desacuerdo & 3 & 7,9 & 7,9 & 94,7 \\
& Muy en desacuerdo & 2 & 5,3 & 5,3 & 100,0 \\
\cline { 2 - 5 } & Total & 38 & 100,0 & 100,0 & \\
\hline
\end{tabular}

Fuente: Encuesta.

En la tabla 3 se aprecia que el 26,3\% está de acuerdo en que el uso de los medios digitales son una herramienta de prevención del acoso escolar y el 42,1\% no está ni de acuerdo ni en desacuerdo lo que abre la posibilidad para aplicar la estrategia del storytelling y cambiar esta percepción de los estudiantes.

\section{PROPUESTA}

Los estudiantes están de acuerdo en que la institución genere un plan sobre el acoso escolar, para esto se propone utilizar una nueva herramienta como estrategia que es el Storytelling la misma que incluye innovadores recursos digitales para su desarrollo y construcción.

La propuesta es que los estudiantes sean los creadores del storytelling utilizando los recursos tecnológicos innovadores relacionadas con el bullying, de esta manera se pretende prevenir actos de acoso escolar entre los estudiantes de la institución para mejorar la convivencia entre la comunidad educativa ya que al momento de crear estos videos el estudiante llegará a interrelacionarse con sus compañeros debido a que se puede trabajar en equipo y profundizar los valores de respeto y empatía para una convivencia armónica. 
La estrategia está realizada en 5 fases: Historia, emociones, autenticidad, simple y conectar emocionalmente con la otra persona a través de una historia.

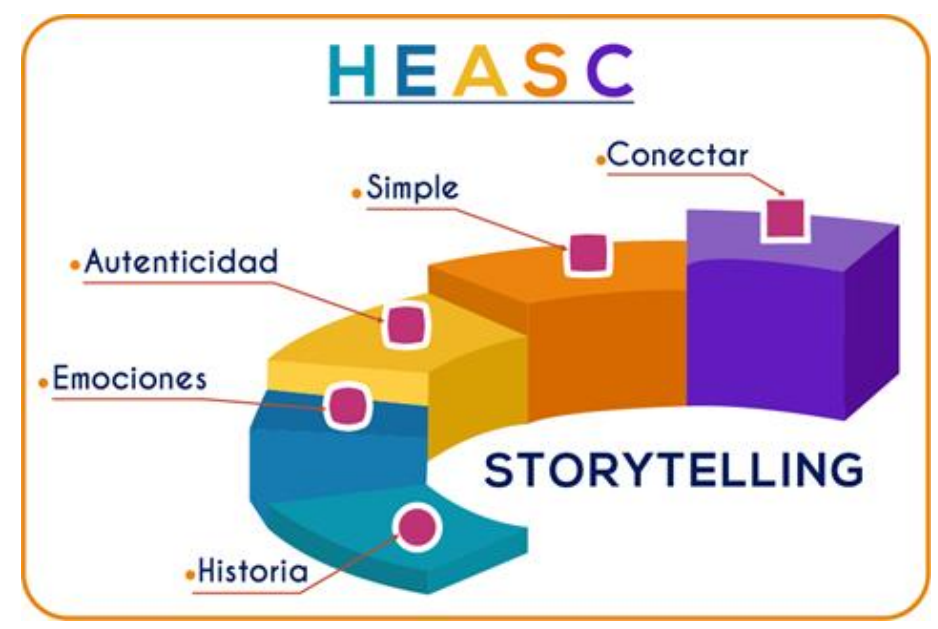

Figura 1. HEASC-Storitelling.

Elaboración: Los autores.

Fase 1: Historia. En esta fase es muy importante pensar que es lo que quiere transmitir, pensar en el mensaje. Al realizar la historia lo que se pretende obtener es una narrativa relacionada con el bullying generando interés en la audiencia, llegando a integrar vivencias en las que se sientan identificadas y de esa manera captar la atención de los espectadores.

Fase 2: Emociones. Aquí lo que se busca es en apelar a las emociones, que el estudiante se integre a la historia como si fuera parte de ella. Con ello conseguimos crear una relación más estrecha evocando momentos vividos, en este caso con el bullying para crear una conexión entre los estudiantes y el interlocutor. 
Fase 3: Autenticidad. Que la historia que realizamos sea verdadera, no copia de alguna otra ni adaptaciones, sino que lo que vamos a contar sea propia de una experiencia. La narrativa contada tiene que tener coherencia, se puede relatar los hechos vividos del bullying para generar un lazo con los espectadores, tener en cuenta que al momento de contar la historia debemos ser nosotros mismos para no caer en otro personaje.

Fase 4: Simple. Todo el contenido tiene que ser simple y corto, lo que se desea transmitir tiene que ser el objetivo principal, una de las cosas más importantes es aprender a contar historias con eficacia. La historia del bullying que vamos a transmitir debe ser muy claro que los estudiantes entiendan que el objetivo principal es la prevención del acoso escolar resaltando los valores para una convivencia armónica.

Fase 5: Conectar. Conectar emocionalmente para que los estudiantes entiendan el mensaje que se quiera dar ya que se vincula fácilmente con un evento similar que hayan vivido. Esto generara en los estudiantes que se identifiquen con la historia similar a la suya obteniendo el resultado deseado que es la prevención del bullying.

\section{CONCLUSIONES}

El tema de la presente investigación que ha sido tratado sirvió como un aporte importante para conocer las necesidades actuales de los estudiantes de la institución educativa American School en el ámbito de la prevención del bullying, debido a que manifiestan que la prevención del acoso escolar debe ser parte del plan de estudios y que las actuales metodologías utilizadas ya no son eficaces para tratar este tema.

Para ello se debe implementar una estrategia diferente a las ya existentes, como el uso del Storytelling mediante la creación de sus propios videos que ayuda a fortalecer los vínculos entre compañeros a desarrollar la creatividad y el trabajo en equipo y de esta 
manera resaltar y promover los valores del respeto, empatía con el propósito de una convivencia armónica entre pares.

Con el fin de ayudar a prevenir el bullying involucrando a los estudinates desde lo emocional y resaltando los valores para una convivencia armonica, deberan los docentes contar con solidos conocimientos en temas de el acoso escolar y storytelling garantizando la implementacion de esta estrategia.

Es importante tener en cuenta que el bullying puede presentarse de diversas formas por medio de insultos o apodos ofensivos, víctima de rumores, quitaron sus pertenencias, agresión mediante medios digitales o cyberbullying, y de carácter físico, pero la que sobresale hoy en día sigue siendo de carácter psicológico y en donde más se produce es durante las clases, por eso es importante que los docentes también estén involucrados para prevenir esta problemática que afecta a varios lugares.

\section{FINANCIAMIENTO}

No monetario.

\section{AGRADECIMIENTO}

A la Universidad Católica de Cuenca; por todo el apoyo brindado en la motivación y desarrollo de esta investigación.

\section{REFERENCIAS CONSULTADAS}

Buenaño-Pico, J. L. (2016). La técnica de narración de cuentos (storytelling technique) y la competencia lectora del idioma inglés de los estudiantes de décimo año de educación general básica de la unidad educativa "Bolívar" de la ciudad de Ambato, provincia de Tungurahua [The storytelling technique and the English language reading competence of tenth year students of basic general education of the educational unit "Bolívar" in the city of Ambato, Tungurahua province]. Recuperado de https://n9.cl/w39re 
Castillo-Pulido, L. (2011). El acoso escolar. De las causas, origen y manifestaciones a la pregunta por el sentido que le otorgan los actores. Magis. Revista Internacional de Investigación en Educación, 4(8),415-428. https://www.redalyc.org/articulo.oa?id=281021722009

Chautard, T., \& Collin-Lachaud, I. (2019). Introducing the storytelling analysis methodology in marketing: Principles, contributions and implementation. Recherche et Applications En Marketing (English Edition), 34(3), 27-46. https://doi.org/10.1177/2051570719841225

Fokides, E. (2017). Using Digital Storytelling to Inform Students About Bullying: Results of a Pilot Program. International Journal of Bias, Identity and Diversities in Education (IJBIDE), 2(1), 27-39. http://doi.org/10.4018/IJBIDE.2017010103

Harris, M. A., \& Orth, U. (2020). The link between self-esteem and social relationships: A meta-analysis of longitudinal studies. Journal of Personality and Social Psychology, 119(6), 1459-1477. https://doi.org/10.1037/pspp0000265

Herrera-López, M., Romera, E., \& Ortega-Ruiz, R. (2018). Bullying y cyberbullying en Latinoamérica. Un estudio bibliométrico [Bullying and cyberbullying in Latin America: A bibliometric study]. Revista Mexicana de Investigación Educativa RMIE, 23, 14056666. https://n9.cl/nc13

Jiang, S., Liu, R.-D., Ding, Y., Jiang, R., Fu, X., \& Hong, W. (2020). Why the Victims of Bullying Are More Likely to Avoid Involvement When Witnessing Bullying Situations: The Role of Bullying Sensitivity and Moral Disengagement. Journal of Interpersonal Violence. https://doi.org/10.1177/0886260520948142

Mathisen, L. (2019). Storytelling: a way for winter adventure guides to manage emotional labour. Scandinavian Journal of Hospitality and Tourism, 19(1), 66-81. https://doi.org/10.1080/15022250.2017.1411827

McLellan, H. (2007). Digital storytelling in higher education. J. Comput. High. Educ. 19, 65-79. https://doi.org/10.1007/BF03033420

Orth, U., \& Robins, R. W. (2014). The Development of Self-Esteem. Current Directions in Psychological Science, 23(5), 381-387. https://doi.org/10.1177/0963721414547414 
Revista Arbitrada Interdisciplinaria KOINONIA

Año VI. Vol VI. N4. Edición Especial: Educación III. 2021

Hecho el depósito de Ley: FA2016000010 ISSN: 2542-3088

FUNDACIÓN KOINONIA (F.K). Santa Ana de Coro. Venezuela.

Miriam Viviana Mora-Bojorque; Luis Bolívar Cabrera-Berrezueta; Nelson Cristóbal Reascos-Vallejo

Roxanna Auccahuallpa-Fernández

Puspitasari, D., Maulida, H., \& Nofiyanto, N. (2018). DST (digital storytelling) to familiarize 'stop bullying' cases among elementary school aged-children. Islamic Studies Journal for Social Transformation, 18, 24. https://doi.org/10.28918/isjoust.v2i1.1610

Tsai MK., Tseng SS., Weng JF. (2011) A Pilot Study of Interactive Storytelling for Bullying Prevention Education. In: Chang M., Hwang WY., Chen MP., Müller W. (eds) Edutainment Technologies. Educational Games and Virtual Reality/Augmented Reality Applications. Edutainment 2011. Lecture Notes in Computer Science, vol 6872. Springer, Berlin, Heidelberg. https://doi.org/10.1007/978-3-642-23456-9 89

Valdés-Cuervo, Á. A., Tánori-Quintana, J., Sotelo-Quiñonez, T. I., \& Ochoa-Arreola, J. A. (2018). Prácticas docentes, clima social, seguridad escolar y violencia entre estudiantes [Teaching practices, climate social, school security and violence between students]. Magis. Revista Internacional de Investigación en Educación, 10(21), 109-120. https://doi.org/10.11144/Javeriana.m10-21.pdcs

Vargas-Martínez, D. E., Jiménez-Jiménez, W. A., \& Duran, J. M. (2018). Acoso escolar y habilidades sociales: aproximación a la problemática [School bullying and social skills: approach to the problem]. http://hdl.handle.net/11371/2723

Wu, J., \& Der-Thanq, V. C. (2020). A systematic review of educational digital storytelling. $\begin{array}{llll}\text { Computers and } & 103786 .\end{array}$ https://doi.org/10.1016/..compedu.2019.103786 